\title{
FINE STRUCTURE IN PLANETARY NEBULAE
}

\author{
YERVANT TERZIAN \\ National Astronomy and Ionosphere Center, Cornell University, Ithaca, N.Y., U.S.A.
}

\begin{abstract}
A short report is presented on the aperture synthesis radio observations of planetary nebulae performed by Terzian et al. (1974). Examples of radio maps at $8085 \mathrm{MHz}$ are shown with angular resolutions of the order of $2^{\prime \prime}$ to $3^{\prime \prime}$.
\end{abstract}

\section{Fine Structure in Planetary Nebulae}

Recently aperture synthesis techniques at centimeter wavelengths have made possible the study of planetary nebulae with angular resolutions as good as 2" (Terzian et al., 1974; Balick et al., 1973; and Scott, 1973). Such observations have in many cases angular resolutions comparable to optical photographs.

In this short report I should like to present the most recent results of aperture synthesis observations which I have completed with the collaboration of B. Balick and C. Bignell (Terzian et al., 1974). Observations were made using the National Radio Astronomy Observatory (NRAO) three-element interferometer at observing frequencies of $2695 \mathrm{MHz}(\lambda 11 \mathrm{~cm})$ and $8085 \mathrm{MHz}(\lambda 3.7 \mathrm{~cm})$. This instrument consists of three 85 -ft $(25.4 \mathrm{~m})$ telescopes, two of which can be moved along a linear track to form up to 16 different telescope separations from 100 to $2700 \mathrm{~m}$.

The synthesis maps of the observed planetary nebulae were generated from the data by the standard Fourier inversion programs available at NRAO. The maps generated in this fashion contain sidelobes because of the unfilled nature of the synthesized aperture. In order to remove the sidelobes, these maps were 'cleaned' using a pattern recognition technique described by Fomalont (1973) and Högbom (1974).

More than 40 planetary nebulae were observed in this program. Complete observations on the available telescope spacings have been performed for 14 nebulae, for which radio maps have been produced. Figures 1 to 4 show examples of radio maps at $8085 \mathrm{MHz}$ for NGC 6543, NGC 7027, NGC 7354 and NGC 7662.

The results of this study show that several of the observed planetary nebulae have a general double source structure - this is more evident in the lower-resolution 2695 MHz maps. The higher-resolution $8085 \mathrm{MHz}$ maps show substantial fine structure. In addition, spherical or elliptical shells are present for most of the observed nebulae, and central intensity depressions appear in the nebulae for which the synthesized beam is smaller than the source dimensions.

NGC 6543, shown in Figure 1, is a helical type nebula studied by Münch (1968) and has a complex optical structure. There exists some correlation between the bright optical features of the nebula and the radio intensity peaks. Woolf (1969) has reported a large infrared excess radiation from NGC 6543.

Figure 2 shows the radio structure of NGC 7027. The optical appearance of this nebula is irregular with bright condensations. Significant local obscuration must exist 


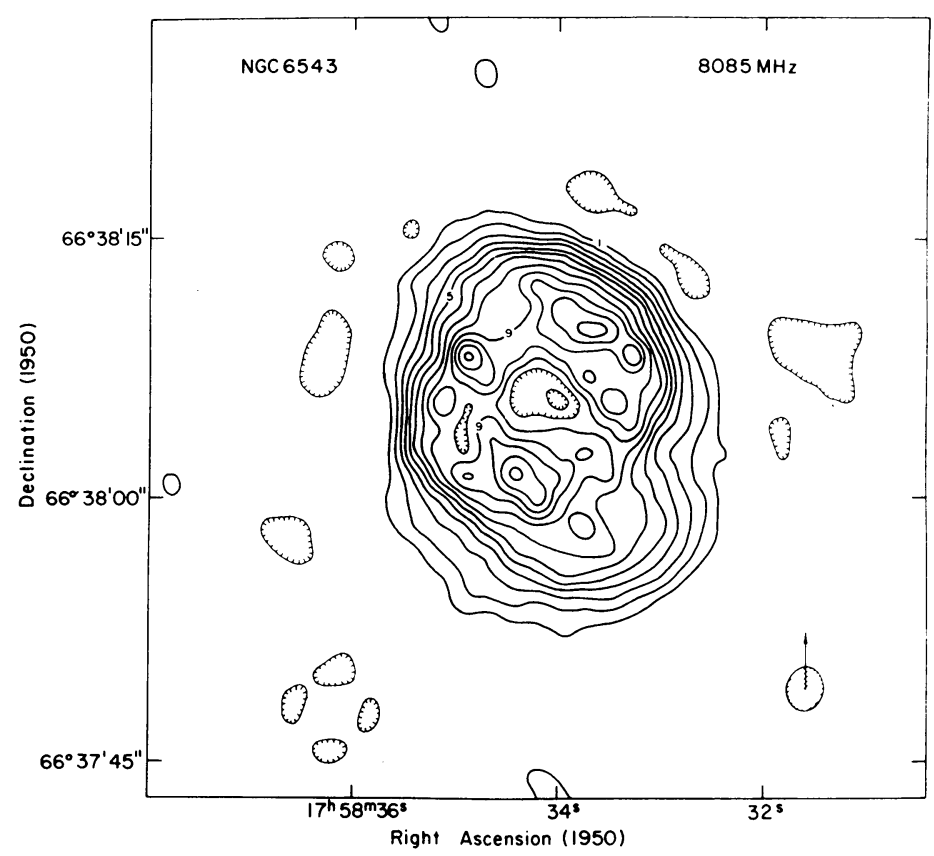

Fig. 1. The radio brightness distribution for NGC 6543 at $8.1 \mathrm{GHz}$. The dimensions of the synthesized beam are $2.5^{\prime \prime} \times 2.2^{\prime \prime}$. The contour spacings correspond to $6.7 \mathrm{~K}$ of brightness temperature.

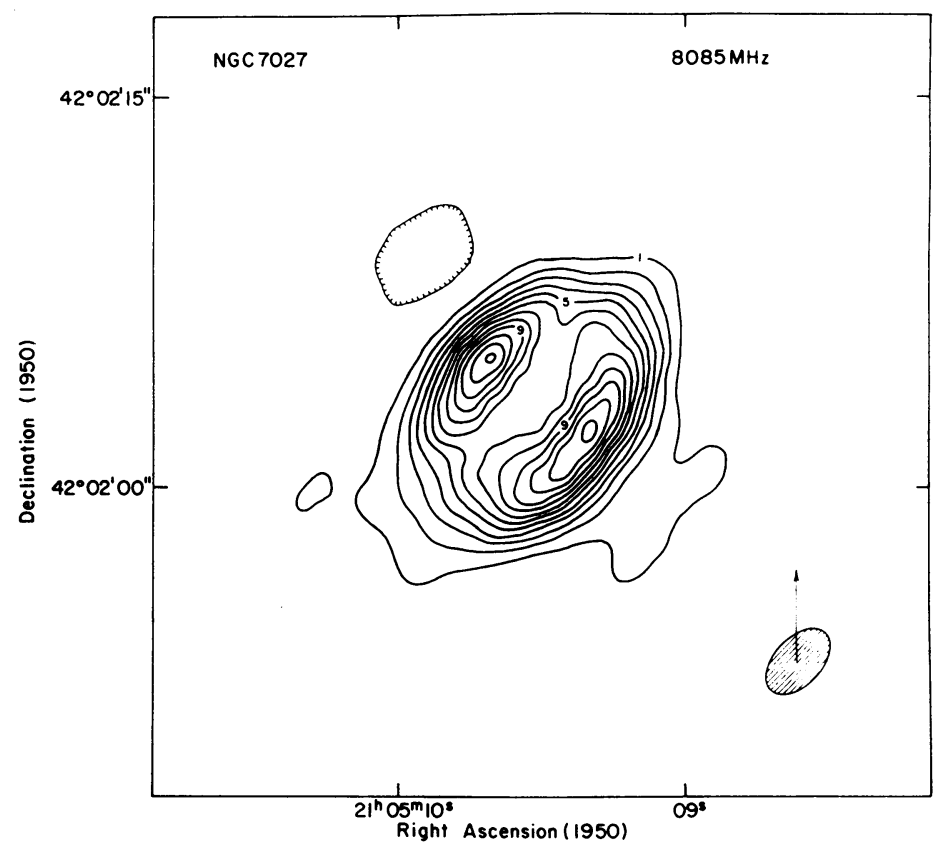

Fig. 2. The radio brightness distribution for NGC 7027 at $8.1 \mathrm{GHz}$. The dimensions of the synthesized beam are $3.0^{\prime \prime} \times 1.9^{\prime \prime}$. The contour spacings correspond to $200 \mathrm{~K}$ of brightness temperature. 


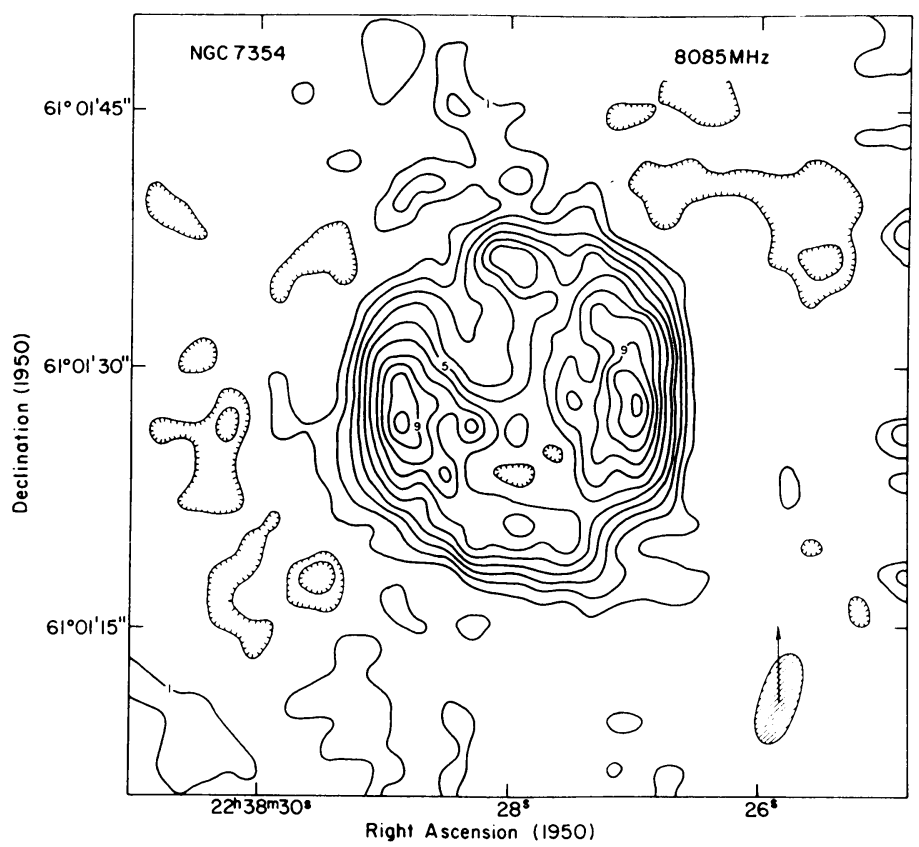

Fig. 3. The radio brightness distribution for NGC 7354 at $8.1 \mathrm{GHz}$. The dimensions of the synthesized beam are $2.7^{\prime \prime} \times 2.1^{\prime \prime}$ (note drawing error in beam major axis). The contour spacings correspond to $2.9 \mathrm{~K}$ of brightness temperature.

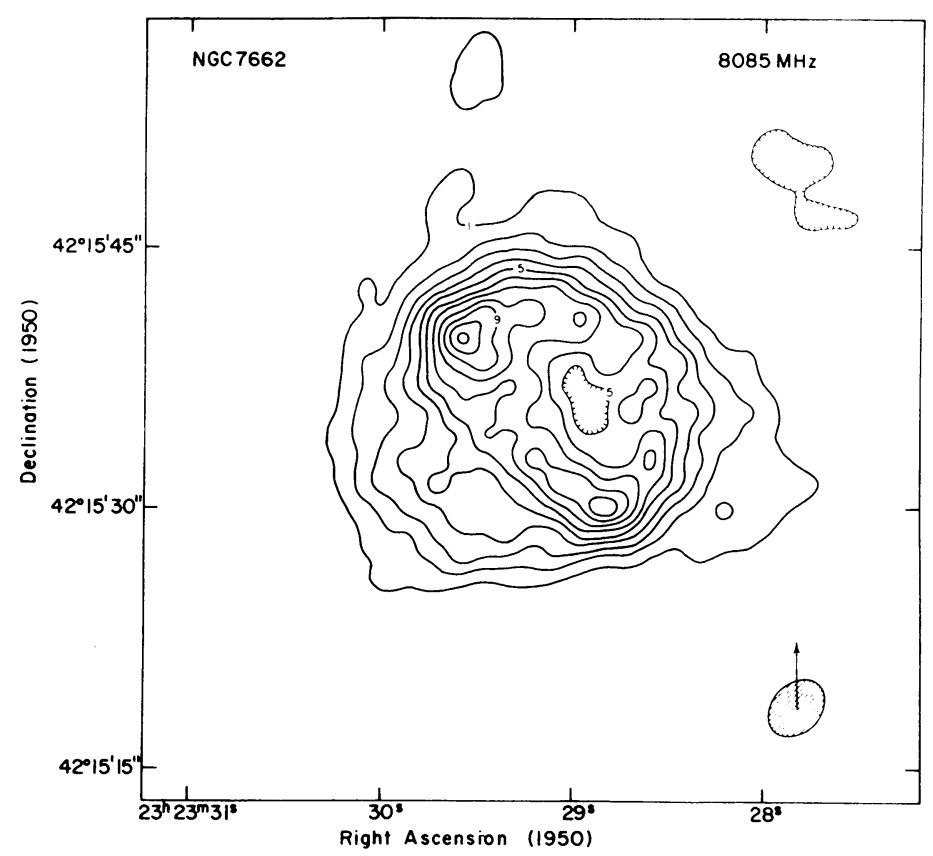

Fig. 4. The radio brightness distribution for NGC 7662 at $8.1 \mathrm{GHz}$. The dimensions of the synthesized beam are $3.5^{\prime \prime} \times 2.0^{\prime \prime}$. The contour spacings correspond to $5.8 \mathrm{~K}$ of brightness temperature. 
in NGC 7027, particularly in the north-east direction. The north-east major radio component is hardly visible at optical wavelengths. It is well known that NGC 7027 has a very large infrared excess (Gillett et al., 1972). Recently Wynn-Williams (1973) has produced an intensity map of NGC 7027 at $\lambda 11 \mu \mathrm{m}$ which greatly resembles the radio map shown in Figure 2.

Optical photographs of NGC 7354 (shown in Figure 3) show that it is composed of an irregular oval ring $22^{\prime \prime} \times 18^{\prime \prime}$ in size, which is surrounded by an outer fainter envelope $\sim 32^{\prime \prime}$ in size. The two main radio peaks have a good correspondence with the optical maxima of the oval ring structure.

Figure 4 shows the radio map for NGC 7662. At optical wavelengths this nebula shows two very thin rings. Aller (1971) has given optical intensity contours superimposed on a photograph of NGC 7662. The $8085 \mathrm{MHz}$ radio map is very similar to these results, indicating no large changes in obscuration across the nebula.

The aperture synthesis radio observations of planetary nebulae give the spacial distribution of matter in these objects, since interstellar extinction and/or local absorption is negligible at radio wavelengths. Such studies should help us understand the origin of planetary nebulae.

\section{Acknowledgements}

The aperture synthesis work was supported by the National Astronomy and Ionosphere Center, which is operated by Cornell University under contract with the National Science Foundation, and by the National Radio Astronomy Observatory, which is operated by Associated Universities, Inc., under contract with the National Science Foundation.

\section{References}

Aller, L. H.: 1971, The Planetary Nebulae, Sky and Telescope Monographic Series I.

Balick, B., Bignell, C., and Terzian, Y.: 1973, Astrophys. J. Letters 182, L117.

Fomalont, E.: 1973, Proc. Inst. Elec. Electron. Engrs. 61, 2111.

Gillett, F. C., Merrill, K. M., and Stein, W. A. : 1972, Astrophys. J. 172, 367.

Högbom, J. A.: 1974, Astron. Astrophys. Suppl. 15, 417.

Münch, G.: 1968, in D. E. Osterbrock and C. R. O'Dell (eds.), 'Planetary Nebulae', IAU Symp. 34, 259.

Scott, P. F.: 1973, Monthly Notices Roy. Astron. Soc. 161, 35.

Terzian, Y., Balick, B., and Bignell, C.: 1974, Astrophys. J. 188, 257.

Wynn-Williams, C. G.: 1973, private communication.

Woolf, N. J.: 1969, Astrophys. J. Letters 157, L37.

Yervant Terzian

National Astronomy and Ionosphere Center,

Cornell University,

Ithaca, N.Y. 14850, U.S.A. 


\section{DISCUSSION}

Shakeshaft: I suggest that it is misleading to describe these radio sources as having mainly double structures, since the features you refer to as double are generally just peaks upon the broader components. The Cambridge $5 \mathrm{~km}$ map of NGC 7027 at $6 \mathrm{~cm}$ has such a double feature, and Paul Scott was able to fit this map very well by a model with a thick cylindrical shell, the axis of which is at about $30^{\circ}$ to the line of sight.

Terzian: Many optical photographs of planetary nebulae show distinct brightness maxima at opposite directions; it is not surprising for the radio observations to suggest a similar pattern. In our work so far we have not made any attempt to fit cylindrical models, mainly because we have seen that at the higher resolutions the sources have a great deal of fine structure and cylindrical models will be unrealistic.

Dickel: If these planetaries really are shells with two more or less symmetrically opposite condensations, is there any correlation of the orientation of these blobs with galactic coordinates, magnetic field, or anything else?

Terzian: I think the number of sources studied in detail are still very small to be able to do such statistical studies.

Wynn-Williams: Observations of NGC 7027 by Becklin, Neugebauer and myself at $10 \mu \mathrm{m}$ with 2 " resolution show that the dust giving rise to the infrared emission has a symmetrical structure similar to that at radio wavelengths. The hot dust is, therefore, almost certainly unrelated to the cold dust causing the extinction seen in part of the source, since the extinction is seen to be so uneven.

Westerhout: Can Shakeshaft explain the physics of a 'cylindrical' shell?

Shakeshaft: I do not know what might be the physical origin of a cylindrical shell, but it is a model that has been used successfully to account for the distribution of optical emission in various planetary nebulae.

Habing: D. George and M. Kaftan-Kassim have observed three planetary nebulae with the Westerbork Synthesis Radio Telescope at $6 \mathrm{~cm}$ with 6" resolution. In the two well resolved cases (NGC 6720 and NGC 40) they find structures that resemble cylindrical models.

Menon: It is rather curious that in the majority of cases you have studied, the planetary nebulae seem to select the orientation of the maximum of the interferometer resolution to show their double structure.

Terzian: This is not so. In the cases where the synthesized beam is nearly circular the orientation of the double structure is random.

Ekers: This effect could be caused by the elliptical cross section of the beam. For example, a ring of emission observed with an elliptical beam will have two peaks in the position angle of the minor axis of the beam. I suggest that you should convolve these maps to a circular beam in order to obtain a better estimate of the brightness distribution.

Terzian: I certainly agree. This will be helpful in the cases where the beam is very elongated.

Milne: If these objects were supernova remnants, we would have no hesitation interpreting these observations in terms of spherical shells.

Zuckerman: Have your results been able to shed any light on the question of recombination lines?

Terzian: I believe only three planetary nebulae show the recombination line so far - NGC 7027, IC 418 . and NGC 6543. The recombination lines in planetary nebulae are exceedingly weak and one has to integrate over $30 \mathrm{~h}$ with the best telescopes in order to get any detection. However, even the few existing results are difficult to explain, and we haven't tried to work out any relationship yet. 